\title{
Analysis of comparative performance of deep learning models for senti- ment analysis
}

\author{
Mirza B. Murtaza \\ Jacksonville University, Jacksonville, FL \\ mmurtaz@ju.edu
}

\begin{abstract}
Sentiment analysis of text can be performed using machine learning and natural language processing methods. However, there is no single tool or method that is effective in all cases. The objective of this research project is to determine the effectiveness of neural network-based architecture to perform sentiment analysis of customer comments and reviews, such as the ones on Amazon site. A typical sentiment analysis process involves text preparation (of acquired content), sentiment detection, sentiment classification and analysis of results. In this research, the objective is to a) identify the best approach for text preparation in a given application (text filtering approach to remove errors in data), and, most importantly, b) what is the best machine learning (feed forward neural nets, convolutional neural nets, Long Short-Term Memory networks) approach that provides best classification accuracy.

In this research, a set of three thousand two hundred reviews of food related products were used to train and experiment with a neural network-based sentiment analysis system. The neural network implementation of six different models provided close to one-hundred percent accuracy of test data, and a decent test accuracy in mid-80\%. The results of the research would be useful to businesses in evaluating customer preferences for products or services.
\end{abstract}

